\title{
One-dimensional $\left(\mathrm{Mo}_{3} \mathrm{~S}_{3}\right)_{n}$ clusters: Building blocks of clusters materials and ideal nanowires for molecular electronics
}

\author{
S. Gemming ${ }^{a}$, G. Seifert ${ }^{\text {b,**}}$, N. Bertram ${ }^{c}$, T. Fischer ${ }^{c}$, M. Götz $^{c}$, G. Ganteför ${ }^{c}$ \\ anstitute of Ion Beam Physics and Materials Research, Forschungszentrum Rossendorf, D-01314 Dresden, Germany \\ b Physikalische Chemie, Technische Universität Dresden, D-01062 Dresden, Germany \\ 'Department of Physics, University of Konstanz, D-78457 Konstanz, Germany
}

\begin{abstract}
A B S T R A C T
The geometric and electronic structures of gas phase $\left(\mathrm{Mo}_{3} \mathrm{~S}_{3}\right)_{n} \mathrm{~S}_{2}$ and $\left(\mathrm{Mo}_{3} \mathrm{~S}_{3}\right)_{n-1}\left(\mathrm{MoS}_{4}\right)_{2}$ clusters with $n=2,3,4, \ldots$ are studied experimentally using mass and photoelectron spectroscopy. The $\mathrm{Mo}_{3} \mathrm{~S}_{3}$ units form one-dimensional chains with length ' $n$ '. There are two possible types of terminations at the ends: a single $\mathrm{S}$ atom or $\mathrm{a} \mathrm{MoS}_{4}$ cluster. The experimental results are compared to calculations based on density-functional theory. Although clusters of this type have been known for decades as constituents of Chevrel phases, we here report their first gas phase synthesis for sizes up to $n=17$. With increasing $n$. the gap vanishes extending such clusters to a conducting wire.
\end{abstract}

\section{Introduction}

Bulk molybdenumdisulfide $\left(\mathrm{MoS}_{2}\right)$ has a certain resemblance to graphite: it consists of weakly bound two-dimensional S-Mo-S layers. And similar to carbon clusters, $\mathrm{Mo}_{n} \mathrm{~S}_{m}$ clusters exhibit a huge variety of geometric structures [1]. Depending on the metal-sulfur ratio there are three-dimensional compact structures $[2,3]$, two-dimensional platelets $[4,5]$, inorganic fullerenes $[6,7]$ and nanotubes [8]. Here, we focus on quasi-one-dimensional structures: $\mathrm{Mo}_{n} \mathrm{~S}_{m}$ nanowires. Such elongated metal-chalcogenide clusters have been known for decades as main constituents of the so-called Chevrel phases $[9,10]$. Those bulk materials consist of negatively charged clusters with the stochiometry $A_{3 n} B_{3 n+2}$ $(A=\mathrm{Mo}, \mathrm{W} ; \mathrm{B}=\mathrm{S}, \mathrm{Se})$ and metal counter ions. The simplest cluster forming a Chevrel phase consists of six metal atoms forming an octahedron and eight sulphur or selenium atoms capping the eight triangular faces. This is a rather stable species and theory predicts that such clusters can form regular arrays on a gold substrate $[11,12]$. By stacking of the planar $A_{3} B_{3}$ units a nanowire can be formed with all metal atoms inside and the outside covered with $S$ or Se atoms. The largest cluster forming a Chevrel phase synthesized up to now is $\mathrm{Mo}_{36} \mathrm{~S}_{38}(n=9)$ with $10 \mathrm{Rb}$ atoms per cluster needed as counter ions [13]. Chevrel clusters can also be synthesized in solution as ligand-stabilized species [14]. Here, even longer chains consisting of $\mathrm{Mo}_{3} \mathrm{~S}_{3}$ units have been synthesized [15]

Surprisingly, there are no reports of such clusters observed as isolated, ligand-free species. This is an unusual situation, because in cluster science many stable species have been identified in gas

\footnotetext{
* Corresponding author.

E-mail address: Gottliard.Seifert@chemie.tu-dresden.de (G. Seifert)
}

phase experiments, and in almost all cases it is unknown whether cluster materials can be synthesized from them. In the case of the Chevrel phases, the situation is inverse: the cluster-containing material is well known, but the gas phase building blocks have so far never been observed in isolated form. As a consequence, the properties of the naked clusters are basically unknown. It was not even clear, whether such nanowires are stable outside a matrix.

Here, we present theoretical and experimental results on the geometric and electronic structure of elongated clusters with a $\left(\mathrm{Mo}_{3} \mathrm{~S}_{3}\right)_{n}$ backbone. Using mass spectroscopy, singly charged Chevrel cluster anions up to $n=17$ are identified and the spectra indicate the existence of even longer wires. Calculations and photoelectron spectra show, that only the smallest clusters have a detectable HOMO-LUMO gap of $0.5-0.9 \mathrm{eV}$, while the larger species can be viewed as metallic conductors. The calculations are extended to very long $\left(\mathrm{Mo}_{3} \mathrm{~S}_{3}\right)_{n}$-based nanowires being promising candidates for components of future nanoelectronic devices [16].

\section{Methods}

The experimental set up is described in detail elsewhere [2-4]. In short, $\mathrm{Mo}_{k} \mathrm{~S}_{1}^{-}$cluster anions are generated using a pulsed arc cluster ion source (PACIS). Molybdenum is evaporated in a pulsed electric arc in a helium atmosphere. A carefully controlled amount of $\mathrm{H}_{2} \mathrm{~S}$ is introduced into the extender. The mixture of Mo clusters, helium carrier gas and $\mathrm{H}_{2} \mathrm{~S}$ is heated up again in an electric discharge downstream in the extender. This results in annealing and produces clusters in their most stable geometries [17]. With the PACIS, sufficient amounts of negatively charged clusters are produced directly. No further charging is necessary. The cluster/carrier 
gas mixture exits the extender forming a supersonic beam and passes through a skimmer and a differential pumping stage. Mass separation is achieved using a high throughput reflectron type time-of-flight mass spectrometer with a resolution of $m / \Delta m=$ 400 . A selected anion bunch can be further examined using photoelectron spectroscopy. The bunch is irradiated with a pulse of an excimer laser with a photon energy of $6.4 \mathrm{eV}$ and the kinetic energy of the detached photoelectron is measured using a magnetic bottle type time-of-flight mass spectrometer.

The present density-functional calculations on the four smallest cluster species were carried out with the programs DEMON [18] and ABINIT [19]; the latter also yielded the properties of the infinitely extended $\left(\mathrm{Mo}_{3} \mathrm{~S}_{3}\right)_{n}$ wire. All structures were optimised at the localdensity level, and refined employing the gradient-corrected PBE functional. Relativistic effective core potentials were employed for Mo and S, but the core was kept small and the semicore $4 \mathrm{~s}$ and $4 p$ states of Mo were explicitly treated in addition to the valence states that comprise $4 \mathrm{~d}$ and $5 \mathrm{~s}$ of Mo and $3 \mathrm{~s}$ and $3 \mathrm{p}$ of $\mathrm{S}$. More extended structures up to $n=8$ were additionally investigated by density-functional-based tight-binding calculations [DFTB]. Further details on the calculation are reported in Ref. [3].

\section{Results and discussion}

\subsection{Calculations}

The model clusters studied here are built from sulphur-bridged molybdenum triangles with composition $\mathrm{Mo}_{3} \mathrm{~S}_{3}$, which are alternatingly stacked to a $\left(\mathrm{Mo}_{3} \mathrm{~S}_{3}\right)_{n}$ cluster core. Two different terminations were investigated: The first variant leads to clusters with the composition $\left(\mathrm{Mo}_{3} \mathrm{~S}_{3}\right)_{n} \mathrm{~S}_{2}$, which is close to the ideal nanowire stoichiometry of Mo:S=1:1. Such clusters are terminated by single sulphur atoms, which are coordinated to the centres of the outermost $\mathrm{Mo}_{3} \mathrm{~S}_{3}$ triangles as depicted in the upper row of Fig. 1, structures (a)-(d). Those clusters are structurally equivalent to the building blocks of solid Chevrel phases. Under more sulphur-rich conditions, i.e., closer to the bulk stoichiometry of $\mathrm{Mo:S}=1: 2$, an alternative termination variant by a tetrahedral $\mathrm{MoS}_{4}$ cap is conceivable, which leads to clusters of the composition $\left(\mathrm{Mo}_{3} \mathrm{~S}_{3}\right)_{n-1}\left(\mathrm{MoS}_{4}\right)_{2}$. As shown in the lower row of Fig. 1, structures (e)-(h), the inner core is again composed of stacked $\mathrm{Mo}_{3} \mathrm{~S}_{3}$ trian-

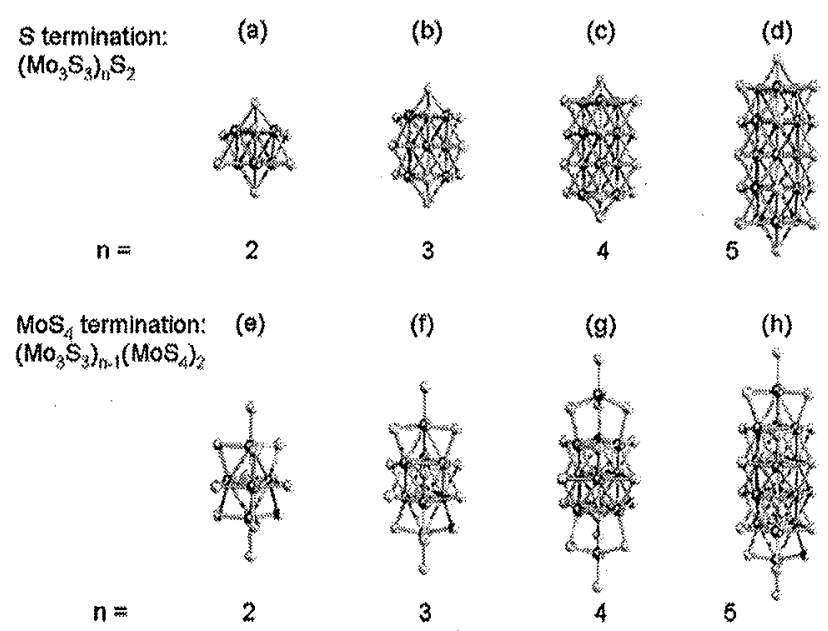

Fig. 1. Schematic representation of the atom arrangement in the investigated clusters. First row depicts clusters terminated by a single $S$ atom: $M_{6} S_{8}(a), M_{5} S_{11}$ (b). $\mathrm{Mo}_{12} \mathrm{~S}_{14}$ (c) and $\mathrm{Mo}_{15} \mathrm{~S}_{17}$ (d); second row gives $\mathrm{MoS}_{4}$-terminated clusters: $\mathrm{Mo}_{5} \mathrm{~S}_{11}(\mathrm{e}), \mathrm{Mo}_{8} \mathrm{~S}_{14}$ (f), $\mathrm{Mo}_{11} \mathrm{~S}_{17}$ (g) and $\mathrm{Mo}_{14} \mathrm{~S}_{20}$ (h) (from left to right, Mo in black, $\mathrm{S}$ in grey). gles, and the $\mathrm{MoS}_{4}$ unit is coordinated to the outermost triangles via three sulphur bridges and saturated by a terminating sulphur atom. As the atomic mass ratio $m(\mathrm{Mo}): m(S)$ is 2.99 , members of the two cluster series with equal values of $n$ have almost identical masses and may occur as overlaid signals in the mass spectrum.

$\mathrm{Mo}_{6} \mathrm{~S}_{8}$, the smallest cluster with termination variant 1 , undergoes a slight Jahn-Teller distortion as neutral species with distances $d(\mathrm{Mo}-\mathrm{Mo})$ between 2.57 and $2.71 \AA$ in the metalloid core and $d(\mathrm{Mo}-\mathrm{S})$ of $2.47 \AA$ to the decorating sulphur atoms. In the anionic species $\mathrm{Mo}_{6} S_{8}^{4-}$ the orbitals responsible for the distortion are fully occupied and the cluster is composed of a perfect central $\mathrm{Mo}_{6}$ octahedron with $d(\mathrm{Mo}-\mathrm{Mo})$ of $2.69 \AA$. which is decorated by the $8 \mathrm{~S}$ atoms at the edge-bridging positions, i.e. in cubic arrangement. For creating the cluster sequence (a)-(d), the $\mathrm{Mo}_{6} \mathrm{~S}_{8}$ cluster is expanded by $\mathrm{Mo}_{3} \mathrm{~S}_{3}$ units along one of the threefold rotation axes of the central octahedron. The resulting Mo-Mo distances expand from an average value of $2.67 \AA$ in $\mathrm{Mo}_{6} \mathrm{~S}_{8}$ to $2.77 \AA$ in the more extended clusters. The Mo-S distances, on the other hand, remain at about $2.49 \AA$.

For termination variant 2 , the smallest cluster, $\mathrm{Mo}_{5} \mathrm{~S}_{11}$, is composed of an elongated, regular trigonal bipyramid, $\mathrm{Mo}_{5}$, with 9 sulphur atoms bridging the edges and two terminal sulphur atoms bonded to the apical Mo atoms. The central $\mathrm{Mo}_{3} \mathrm{~S}_{3}$ unit is an equilateral triangle with $d(\mathrm{Mo}-\mathrm{Mo})=2.62 \hat{\mathrm{A}}$ and sulphur bridges of $d(\mathrm{Mo}-\mathrm{S})=2.42 \AA$; the Mo-S bonds to the terminating $\mathrm{MoS}_{4}$ fragment amount to $d(\mathrm{Mo}-\mathrm{S})=2.45 \AA$, within the termination unit distances of $d(\mathrm{Mo}-\mathrm{S})=2.30 \AA$ and $d(\mathrm{Mo}=S)=2.16 \AA$ occur. Towards more elongated clusters, the Mo-Mo distances of the cluster core expand in an analogous manner as obtained for termination 1 , while the distances to and within the termination unit do not change significantly.

For reference, the infinitely long $\left[\mathrm{Mo}_{6} \mathrm{~S}_{6}\right]_{\infty}$ wire was investigated by DFT band-structure calculations, employing periodic boundary conditions. The most stable structure is composed of $\mathrm{Mo}_{3} \mathrm{~S}_{3}$ triangles arranged as a staggered stack, with a length of the repeat unit of $4.36 \AA$ and with bond lengths $d(\mathrm{Mo}-\mathrm{Mo})$ of $2.74 \AA$ and $d(\mathrm{Mo}-\mathrm{S})$ of $2.48 \AA$. Other periodic structures like eclipsed arrangements of the $\mathrm{Mo}_{3} \mathrm{~S}_{3}$ triangles are less stable by $0.83 \mathrm{eV} /$ at and more.

All clusters are stable against decomposition into the quite unfavourable capping fragments and the infinitely long wire with binding energies of up to $-0.16 \mathrm{eV} / \mathrm{at}$ for $\mathrm{Mo}_{6} \mathrm{~S}_{8}$ and of $-0.14 \mathrm{eV} / \mathrm{at}$ for $\mathrm{Mo}_{5} \mathrm{~S}_{11}$, and monotonously decreasing values for the longer clusters. The decomposition into free, neutral $\mathrm{Mo}_{3} \mathrm{~S}_{3}$ units and the termination units is even less favourable by up to $1.66 \mathrm{eV} /$ at for termination 1 and by up to $1.58 \mathrm{eV} /$ at for termination 2.

The different stoichiometries make it difficult to compare the stabilities of the two cluster series directly by comparing total energies. However, relative stabilities can be evaluated by a grand canonical approach. For this purpose the total energy per atom, $E_{\text {at }}$, is plotted as a function of the chemical composition, here the mole fraction of molybdenum, $x(\mathrm{Mo})$ (see Fig. 2). All structures are sulphur-rich with $x(\mathrm{Mo})$ values between 0.3 and 0.5 . Clusters with the more sulphur-rich $\mathrm{MoS}_{4}$ termination lead to entries (squares) at lower $x(\mathrm{Mo})$ values than clusters terminated by a single sulphur atom (circles), and smaller clusters contain a higher mole fraction of sulphur than the longer ones. In the sulphur-rich regime with $x(\mathrm{Mo})<0.4$, the $\mathrm{MoS}_{4}$-terminated structures are energetically preferred, whereas S-terminated clusters become stable above that threshold. The dashed and dotted linear regression lines suggest that the crossover between the two termination types occurs at $x(\mathrm{Mo}) \sim 0.46$, but the actual values indicate that both variants are already equally likely to occur from $x(\mathrm{Mo}) \sim 0.4 \mathrm{on}$. Fig. 2 also suggests that on confining the Mo content experimentally to a range of e.g. $0.38<x(\mathrm{Mo})<0.44$, the two smallest clusters would 


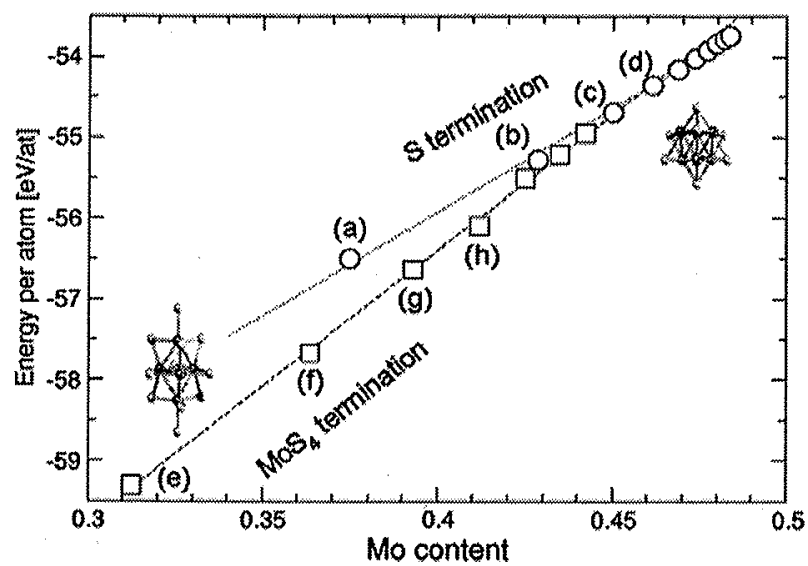

Fig. 2. Calculated energy per atom $E_{\text {at }}$ (in eV/at) as function of the Mo content $x$. Sterminated clusters (a)-(d) and higher homologues are represented by circles, the more S-rich $\mathrm{MoS}_{4}$-terminated wires (e)-(h) and higher homologues are indicated by squares. Dotted and dashed regression lines are added to determine the relative stabilities of S-terminated and $\mathrm{MoS}_{4}$-terminated species, respectively.

be structures (a) and (b) with termination 2, whereas the larger species $(\mathrm{g})$, (h) and higher ones would additionally exhibit termination 2. Exclusively longer clusters might be obtained by choosing an effective Mo:S ratio of close to $1: 1$ in the reactive gas phase.

In addition, the electronic structures of the four smallest structures were investigated theoretically, in order to elucidate details that may help to differentiate between the two termination types. Table 1 summarizes the findings on characteristic energy differences calculated for neutral and charged species. Column two gives the energy gaps between the highest occupied and the lowest unoccupied molecular orbitals evaluated as difference of the corresponding Kohn-Sham eigenvalues of the neutral clusters. Only the smallest clusters $\mathrm{Mo}_{6} \mathrm{~S}_{8}$ and $\mathrm{Mo}_{5} \mathrm{~S}_{11}$ exhibit sizeable HOMO-LUMO gaps of 0.9 and $0.5 \mathrm{eV}$. With increasing cluster extension the HOMO-LUMO gap tends to smaller values with a slight evenodd oscillation, and the ideal, infinitely long wire is metallic with two bands crossing the Fermi level along the $\Gamma=Z$ direction $\left(k_{z}=0.0-0.5\right)$ [20]. A gap can, however, be opened by an elongation or torsion of the extended wire, thus, external stress can modify the electronic properties of the wire $[22,23]$. For the three smallest clusters of both termination variants, larger band gaps of up to $1.4 \mathrm{eV}$ can be obtained by adding negative charges, whereas the larger and more elongated clusters from $n=4$ on would require a higher anionic charge for a similar stabilisation of the electronic structure. Especially for the fourfold negative cluster anions the next larger energy gap between the HOMO +2 and the HOMO +3 is reached for most of the structures.

\section{Table 1}

Calculated HOMO-LUMO gaps (HL) of the neutral clusters, vertical (VDE) and adiabatic (ADE) detachment energies, and vertical (VIP) and adiabatic (AIP) ionization potentials (all energies in $\mathrm{eV}$ ).

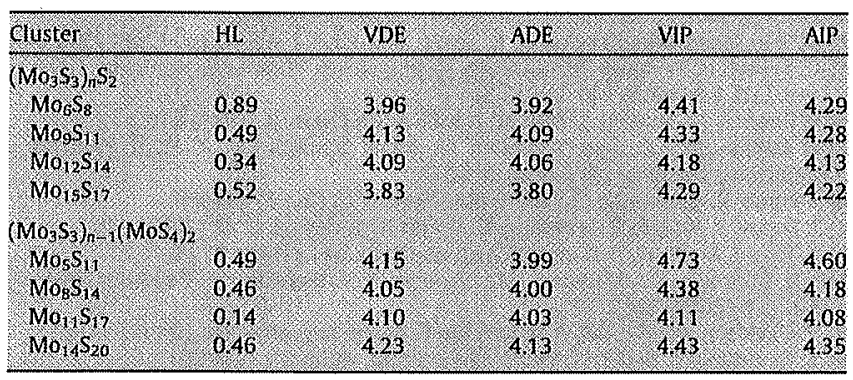

Columns three to six of Table 1 give the vertical and adiabatic detachment and ionization energies as total energy differences between the charged and neutral species ( $\triangle S C F$ procedure). Adiabatic processes rely on a full geometry optimization of all involved species, for the vertical processes the geometry of the initial state is optimized and maintained in the final state. Clusters with termination 2 show a clear trend to larger energies for the detachment of an electron from the cluster anion, indicating a gradual stabilisation of the anion; for the less sulphur-rich clusters, such a trend is obtained only for longer species. Concomitant with previous cluster size studies on homonuclear species, the ionization potentials tend to lower values with growing cluster size [21]. As for the HOMO-LUMO gaps, the trend obtained for the detachment and ionization energies is overlaid by a slight even-odd oscillation, which is also reproduced in DFTB calculations of the more extended species.

\subsection{Experiments}

Fig. 3 displays a mass spectrum of annealed $\mathrm{Mo}_{k} \mathrm{~S}_{l}^{-}$cluster anions. The spectrum is dominated by a progression of outstanding lines starting at a mass corresponding to $\mathrm{Mo}_{6} \mathrm{~S}_{8}^{-}$or $\mathrm{Mo}_{5} \mathrm{~S}_{11}^{-}$ $(n=2)$. The progression follows the $\mathrm{Mo}_{3 n} S_{3 n+2}$ pattern known from the Chevrel phases and can be distinguished from the continuous background up to $n=17$ corresponding to the mass of $\mathrm{Mo}_{51} \mathrm{~S}_{53}^{-}$. However, the mass of three sulphur atoms equals the one of one Mo atom. Accordingly, based on the mass spectra alone it is not possible to decide, whether the progression should be assigned to the $\left(\mathrm{Mo}_{3} \mathrm{~S}_{3}\right)_{n} \mathrm{~S}_{2}$ or the $\left(\mathrm{Mo}_{3} \mathrm{~S}_{3}\right)_{n-1}\left(\mathrm{MoS}_{4}\right)_{2}$ series. With further increasing $n$ the progression submerges in the more or less continuous cluster ion signal caused by the presence of other $\mathrm{Mo}_{k} \mathrm{~S}_{l}^{-}$cluster anions. Probably, for these larger clusters the differences in stability between the more stable Chevrel species and the various other clusters is too small to result in enhanced intensity after the annealing.

Fig. 4 displays photoelectron spectra of the first four members of the series with $n=2-5$ recorded with a photon energy of $6.4 \mathrm{eV}$. The spectrum of the smallest cluster with $n=2$ is different from the larger ones, because a small peak appears at low binding energy. Such a peak indicates the existence of a HOMO-LUMO gap of the neutral cluster and from the spectrum the size of this gap

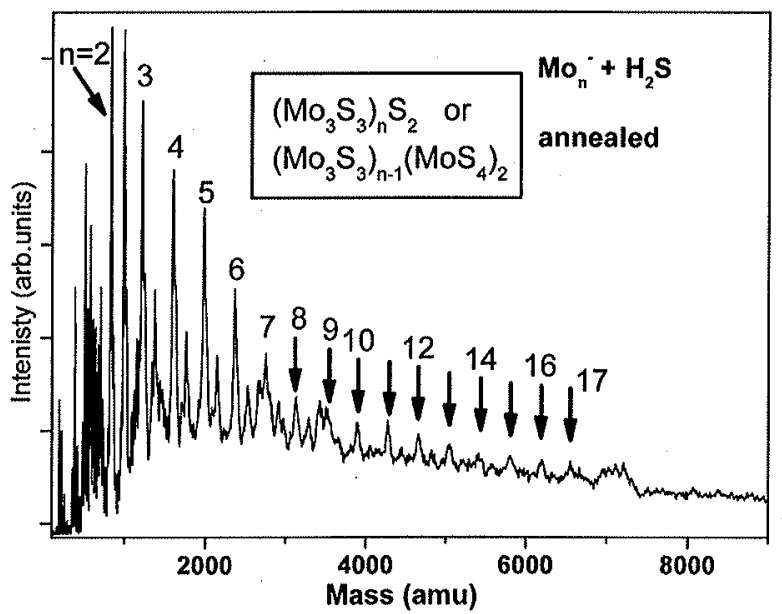

Fig. 3. Mass spectrum of $\mathrm{Mo}_{k} \mathrm{~S}_{k}^{-}$clusters generated using a pulsed arc cluster ion source. In the extender of the source, Mo- cluster anions react with $\mathrm{H}_{2} \mathrm{~S}$ gas. Subsequent annealing at the correct metal-sulphur ratio results in formation of Chevrel cluster anions marked by arrows) with $n=2-17$. Beyond $n=17$, the peaks can no longer be distinguished from background signal of various other $\mathrm{Mo}_{k} \mathrm{~S}_{k}^{-}$ clusters. 


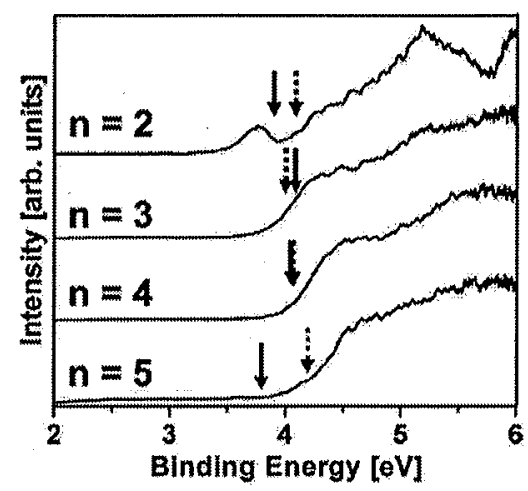

Fig. 4. Photoelectron spectra of $\mathrm{M}_{3 n} \mathrm{~S}_{3 n+2}$ cluster anions. The vertical detachment energy taken as the onset of the electron signal, increases with increasing $n$. Calculated VDE values for $\mathrm{S}$-terminated and $\mathrm{MoS}_{4}$-terminated clusters are indicated by black and white marks, respectively, $A$ small peak at low binding energy is observed for the smallest cluster only indicating the existence of HOMO-LUMO gap of $0.5 \mathrm{eV}$.

can be estimated to be $0.5 \pm 0.15 \mathrm{eV}$. The three larger clusters show an increase of the electron signal at the emission threshold and indicate a small $(<0.2 \mathrm{eV})$ or vanishing gap size. The position of the peak at lowest binding energy (uppermost trace in Fig. 4) can be compared to the calculated vertical detachment energy (VDE, see Table 1). For the other spectra there is no peak at threshold, but a roughly step-like increase of intensity. We compare the calculated VDEs to the binding energies, at which the intensity reaches $50 \%$ of this step-like increase.

\subsection{Comparison between theory and experiment}

First of all, the mass spectrum displayed in Fig. 3 shows, that gas phase Chevrel clusters consisting of stacks of $\mathrm{Mo}_{3} \mathrm{~S}_{3}$ units can be generated as singly charged anions. They are more stable than other $\mathrm{Mo}_{k} \mathrm{~S}_{l}^{-}$cluster anions, because after the annealing the Chevrel clusters appear as outstanding mass peaks [17]. No even/odd alternation of the mass intensities could be identified. A stability alternation such as the one predicted for the neutral clusters might be too small to influence the relative intensities in the mass spectrum.

Based on the mass spectrum alone it is not possible to distinguish between the two possible variants predicted by theory. However, the two versions differ by their electronic structures and a comparison of the measured photoelectron spectra with the calculated vertical detachment energies and the HOMO-LUMO gaps might allow an unambiguous assignment. For variant 1 , the smallest species with $n=2$ has a considerably larger gap than all the other ones, while for variant 2 the $n=2$ cluster has a gap similar to the one of $n=3$ and 5 (Table 1). The experimental photoelectron spectra indicate a considerable gap for $n=2$ and non-detectable gaps for the larger clusters. This difference between the smallest cluster and the larger members of the series hints towards variant 1 (the regular Chevrel clusters) being the one produced in the experiment. The gap of $\mathrm{Mo}_{6} \mathrm{~S}_{8}$ predicted by theory is $0.89 \mathrm{eV}$, while the experimental value is $0.5 \pm 0.15 \mathrm{eV}$. Theory predicts for both versions of the $n=5$ cluster gaps of $0.5 \mathrm{eV}$ (Table 1 ), but the photoelectron spectrum does not indicate the existence of a gap (lowest trace in Fig. 4). This could be explained by the assumption, that theory generally overestimates the gap sizes by 0.3 or $0.4 \mathrm{eV}$. In addition, in the experiment gaps smaller than $0.2-0.3 \mathrm{eV}$ might not be observable due to the widths of the peaks in the photoelectron spectra. With these two assumptions the experimental and theoretical data agree considerably and indicate that at least the smallest cluster is the 'magic' $\mathrm{Mo}_{6} \mathrm{~S}_{8}^{-}$anion. This assignment is supported by the calculated VDE (black arrow in Fig. 4), which agrees within $0.2 \mathrm{eV}$ with the binding energy of the small peak in the upper trace of Fig. 4 . For $n=3$ and 4 the calculated VDEs are similar for both versions and agree almost perfectly with the experimental data (Fig. 4). Here, we cannot distinguish between the two versions. For $n=5$, the VDEs are quite different again and in this case the experiment agrees better with the VDE calculated for termination 2 (lowest trace and dotted arrow in Fig. 4). In general, the method we used for the generation of the cluster anions yields clusters with high electron affinities, which explains the observation of termination 2 for the $n=5$ cluster. For the smallest species, the cluster with the lower VDE (termination 1) is observed. This can be explained by the sulphur-metal ratio. For the generation of larger $\mathrm{Mo}_{3} \mathrm{~S}_{3}$-species the ratio between metal and sulphur needs to be close to $1: 1$. The smallest member of the variant 2 is $\mathrm{Mo}_{5} \mathrm{~S}_{11}$ with a ratio close to $1: 2$. It will not be observed at conditions, when larger Chevrel-like clusters are produced simultaneously like in our experimental set up.

\section{Conclusion}

Density-functional-based calculations and mass and photoelectron spectroscopy experiments were carried out in parallel to elucidate the geometric and electronic structures of gas phase $\left(\mathrm{Mo}_{3} \mathrm{~S}_{3}\right)_{n}$ clusters with $n \leqslant 17$. The main structural motif of those gas phase clusters is very similar to the well-characterized crystal structure of Chevrel phases. The smallest member of the cluster family is $\mathrm{Mo}_{6} \mathrm{~S}_{8}$ with a HOMO-LUMO gap of about $0.5 \mathrm{eV}$. With increasing $n$, the clusters develop into one-dimensional nanowires with a vanishing gap and a metallic-like conductivity along the structure. As the smallest member of the cluster family bonds strongly to gold surfaces [1,11], such extended, metallic-like Chevrel clusters may also readily bond to gold electrodes via the capping sulfur atom at each end of the cluster. Thus, the controllable stoichiometry, the propensity to bond to gold, and the tunable one-dimensionally metallic behavior of the molybdenum core inside the insulating sulfur shell make these clusters promising components of future nano-scale electronic devices.

\section{Acknowledgements}

S.G. and G.S. acknowledge funding from the DFG via SPP 1153 and from the German-Israel Foundation. N.B., T.F., M.G. and G.G. acknowledge the support from the DFG (Deutsche Forschungsgemeinschaft).

\section{References}

[1] A.N. Enyashin, S. Gemming, G. Seifert, Eur. Phys. J. - S. T. 149 (2007) 103.

(2) N. Bertram, Y.D. Kim, G. Ganteför, Q. Sun, P. Jena, J. Tamuliene, G. Seifert, Chem. Pliys. Lett. 396 (2004) 341.

[3] S. Gemming, J. Tamuliene, G. Seifert, N. Bertram, Y.D. Kim, G. Ganteför, Appl Pliys. A 82 (2006).

[4] N. Bertram, J. Cordes, Y.D. Kim, S. Gemming, G. Seifert, G. Ganteför, Chem. Phys. Lett. 418 (2006) 36.

[5] S. Gemming, G. Seifert, Nat, Nanotechnol. 2 (2007) 21

[6] A.N. Enyashin et al., Angew. Chem., Intl. Ed. 46 (2007) 623.

[7] M. Bar-Sadan, l. Kaplan-Ashiri, R. Tenne, Eur. Phys. J. - S. T. 149 (2007) 71

[8] G. Seifert, H. Terrones, M. Terrones, G. Jungnickel, Thomas Frauenheim, Phys. Rev. Lett. $85(2000) 146$.

[9] R. Chevrel, M. Sergent. J. Prigent, J. Solid State Chem. 3 (1971) 515.

[10] E. Kaldis (Ed.), Current Topics in Materials Science, vol. 3, North Holland Publishing Company, Amsterdam, 1979.

[11] I. Popov, S. Gemming, G. Seifert, Phys. Rev. B 75 (2007) 245436.

[12] I. Popov, T. Kunze, S. Gemming, G. Seifert, Eur. Phys. J. D 45 (2007) 439.

[13] S. Picard, M. Potel, P. Gougeon, Angew. Chem. 111 (1999) 2148.

[14] Y.V. Mironov, A.V. Virovets, N.G. Naumev, V.N. Ikorskii, V.E. Fedorov, Chem. Eur. J. 6 (2000) 1361.

[15] P. Davidson, J.C. Gabriel, A.M. Levelut, P. Batail, Europhys. Lett. 21 (1993) 317. [16] S. Gemming, G. Seifert, I. Vilfan, Phys. Status Solidi B 1 (2006). 
[17] H. Kietzmann, R. Rochow, G. Ganteför, W. Eberhardt, K. Vietze, G. Seifert, P.W Fowler, Phys. Rev. Lett. 81 (1998) 5378.

18] A.M. Köster et al., Demon, NRC, Canada, 2004.

[19] The Asinit Code is a Common Project of the Universite Catholique de Louvain Corning Inc., and other contributors.

[20] J.M. Tarascon, F.J. DiSalvo, J.V. Warszczak, Solid State Commun. 52 (1984) 227.
[21] M. Seidl, K.-H. Meiwes-Broer, M. Brack, J. Chem. Phys. 95 (1991) 1295.

[22] J. Kibsgaard, A. Tuxen, M. Levisen, E. Legsgaard, S. Gemming, G. Seifert, Nanoletters 8 (2008) 3928

[23] I. Popov, S. Gemming, S. Okano, N. Ranjan, G. Seifert, NanoLetters 8 (2008) 3928. 